\title{
Luego de un año de la implantación de stents liberadores de drogas no hubo diferencias entre la antiagregación simple o doble
}

After a year of drug-eluting stent implantation there were no differences between simple and dual antiplatelet therapy

Park SJ, col. N Engl J Med 2010;362:1374-82.

\section{Objetivos}

Comparar la eficacia de aspirina (AAS) -simple antiagregacióncontra AAS más clopidogrel -doble antiagregación- en la ocurrencia de infarto de miocardio (IM) o muerte de causa cardiovascular (CV) en pacientes con stents liberadores de drogas a partir de los 12 meses de su implantación.

\section{Diseño}

Se analizaron los datos provenientes de dos ensayos clínicos aleatorizados controlados no ciegos en los que se comparo el uso de simple versus doble antiagregación.

\section{Lugar}

Estudio multicéntrico realizado en 22 centros cardiovasculares de Corea del Sur.

\section{Pacientes}

2.701 pacientes a quienes se les implanto un stent liberador de drogas 12 meses atrás y que durante ese tiempo recibieron doble antiagregación. Estos pacientes debían haber permanecido libres de eventos cardio y cerebrovasculares mayores o de eventos de sangrado mayor. Se excluyeron aquellos pacientes con contraindicaciones para el uso de antiagregantes, o con problemas vasculares concomitantes que requiriesen la continuidad en el uso de clopidogrel.

\section{Intervención}

Se aleatorizó a los pacientes para recibir AAS 100 a $200 \mathrm{mg} / \mathrm{dia}$ más clopidogrel $75 \mathrm{mg} / \mathrm{dia}(\mathrm{n}=1.357)$ o AAS $325 \mathrm{mg} / \mathrm{dia}(\mathrm{n}=1.344)$.

\section{Medición de resultados principales}

El resultado principal se definió como IM o muerte CV (resultado combinado). El análisis fue hecho por intención de tratar*.

\section{Resultados principales}

La media de seguimiento fue de 19,2 meses. Hubo un aumento no significativo de IM y muerte CV en el grupo de doble antiagregación (ver tabla 1).

Tabla 1: Comparación de riesgo acumulado de eventos vasculares a 24 meses en el grupo tratado con AAS vs. AAS más clopidogrel.

\begin{tabular}{c|c|c|c} 
Resultado & $\begin{array}{c}\text { Clopidogrel + } \\
\text { AAS (\%) }\end{array}$ & AAS (\%) & Hazard Ratio (IC 95\%) \\
\hline IM o Muerte CV & 1,8 & 1,2 & $1,65(0,80$ a 3,36) \\
\hline
\end{tabular}

\section{Conclusiones}

El uso de doble antiagregación durante un periodo mayor a 12 meses no fue más efectivo que la simple antiagregación con AAS en reducir la ocurrencia de IM o muerte de causa cardiovascular. Estos hallazgos deberán ser confirmados o refutados mediante ensayos clínicos aleatorizados mas grandes.

Fuente de financiamiento: no informada.

\section{Comentario}

Existen varios estudios actualmente que intentan evaluar tanto la combinación como la duración óptima de la antiagregación en los pacientes que se colocan stents ${ }^{1}$.

Si bien las intervenciones basales fueron similares en ambos grupos, no se reporto si hubo otras co-intervenciones a lo largo del estudio. Consideramos que la presentación de incidencia acumulada* no es la mejor forma de reportar los resultados en este estudio puesto que los eventos son tiempo dependiente y cada participante tuvo un periodo de seguimiento diferente, y en algunos casos, insuficiente. Hubiera sido más deseable la presentación de los mismos como tasa de incidencia*.

Otro punto a considerar y valioso de aclarar es que a pesar de que el estudio no fue ciego, los resultados evaluados son tan objetivos que no hace falta el enmascaramiento en la intervención, ya que la asignación de la causa de muerte si fue enmascarada.
Por último, es importante mencionar que el estudio no logro el poder* esperado para poder tomar conclusiones firmes. Esto se debió básicamente a que hubo menos eventos a los esperados al momento del diseño del estudio.

\section{Conclusiones del comentador}

El estudio no tuvo poder suficiente para responder la pregunta inicialmente planteada, no pudiéndose establecer si existen o no dife-rencias significativas entre los grupos estudiados. Es importante esperar estudios de mayor envergadura. Hasta que esto ocurra, pareciera más prudente la antiagregación simple que la doble. Puesto que la probabilidad de eventos es mucho mayor con la última.

Ignacio Ricci [ Servicio de Medicina Familiar del Hospital Italiano de Buenos Aires. ricardo.ricci@ hospitalitaliano.org.ar ]

Ricci I. Luego de un año de la implantación de stents liberadores de drogas no hubo diferencias entre la antiagregación simple o doble. Evid Act Pract Ambul Oct-Dic 2011;14(4):133. Comentado de: Park SJ, Park DW Kim YH y col. Duration of Dual Antiplatelet Therapy after Implantation of Drug-Eluting Stents. N Engl J Med 2010;362:1374-82.

Referencias

1.Cutlip D, Levin T. Antithrombotic therapy for intracoronary stent implantation: General use. In: UpToDate 19.2, Basow, DS (Ed), UpToDate, Waltham, MA, 2011. 